\title{
An Efficient Iterative Thresholding Method for Compressed Sensing
}

\author{
Amin Tavakoli and Ali Pourmohammad, Member, IACSIT
}

\begin{abstract}
Compressed sensing is one of the newest problems in signal processing area. Recently greedy algorithms, such as matching pursuit (MP), orthogonal matching pursuit (OMP), iterative hard thresholding (IHT) and so on, are become applicable methods for compressed sensing problem. In this paper we propose an efficient technique for thresholding in IHT method. Proposed method simulation results in comparison with OMP and IHT methods, lead us to faster reconstruction and same SNR for reconstructed signal.
\end{abstract}

Index Terms-Compressed sensing, iterative hard thresholding, matching pursuit, orthogonal matching pursuit.

\section{INTRODUCTION}

According to Shannon Nyquist theorem in fidelity signal reconstruction, the sampling rate of signal must be at least twice of highest frequency present in signal. This often results in too many samples and high memory. In real world the most of signals have sparse coefficient or good estimated with sparse coefficient in some orthonormal basis, wavelet, FFT, curvelet, and so on. Compressed Sensing (CS) [1] [2] seeks to represent a signal using a number of linear, non-adaptive measurements and using sparse property to combine acquisition and compression into one step and compress the signal at the time of sampling. In CS we need far fewer samples than Nyquist rate to recover original signal. Recent years many different methods have been proposed to reconstruct signal in CS problem. The two most common approaches are greedy [3] and convex relaxation methods [4]. Iterative greedy algorithm reconstructs signal one step at a time by selecting the atom best correlated with the residual part of the signal and uses it to update the current approximation. Then it produces a new approximant by projecting the signal onto the dictionary elements that have already been selected [5]. Basis pursuit (BP) finds signal representations in overcomplete dictionaries by convex optimization. It obtains the decomposition that minimizes the L1-norm of the coefficients occurring in the representation. Due to depending of it to global optimization, it could stably superresolve in ways which OMP cannot. But the major advantage of OMP is that it admits simple and fast implementation [6] [7]. This paper is organized as follows: Section II introduces the formulation of CS problem. Section III, we introduce the necessary notations, including the OMP, IHT algorithms. Then we present our main result concerning IHT and OMP. Section IV presents an efficient thresholding

Manuscript received February 21, 2012; revised May 27, 2012.

The authors are with the Electrical Engineering Department, Amirkabir University of Technology, Hafez Ave., Tehran 15914, Iran (e-mails: amintavakoli61@gmail.com, pourmohammad@aut.ac.ir, tel.: +98 21 64543392, fax: +982166406469). to improve IHT complexity and archive faster algorithm for large scale signal. Simulation results are discussed in section V. Finally, Section VI makes conclusions.

\section{CS PROBLEM}

The following notations will be used in this paper. Variable $\mathrm{y}$ is a d-dimensional real or complex vector. Variable $\mathrm{x}$ is a n-dimensional real or complex vector. Matrix $\Phi$ is a $\mathrm{d} \times \mathrm{n}$ real or complex matrix whose transpose (hermitian transpose) is $\Phi^{T}$.

Convex problem, such as Basis Pursuit (BP) problem is tabloid in finding least of L1-norm solution. Underdetermined linear system y which is $\mathrm{y}=\Phi \times \mathrm{x}$ [8], can be approximate using $\mathrm{BP}$ as:

$$
\text { minimize }\|b\|_{1} \text { subject to } \Phi \times b=y \quad(B P)
$$

Despite this difficulty, it is shown in [1] that under certain conditions, the sparse representation can be accurately reconstructed using non-adaptive linear measurements which it is formulated as O. $(d \times \log (n))$.

\section{GREEDY ALGORITHM}

Greedy methods are other set of algorithms which they are used for efficiently reconstruction of signals from compressed sensing observations. Two IHT and OMP algorithms of this set are explain as follow:

\section{A. IHT Algorithm}

In [9], IHT algorithm is used to solve the $\mathrm{k}$ sparse problem. Let $b^{0}=0$, IHT algorithm can write as a follow iterative function:

$$
b^{n+1}=H_{k}\left[b^{n}+\Phi^{T}\left(y+\Phi \times b^{n}\right)\right]
$$

where $b^{n+1}$ is reconstructed signal after n-time iteration. Also $H_{k}(x)$ is the non-linear operator which sets all variables except sets the largest (in magnitude) k elements of $x$ to zero. The convergence of this algorithm was proven in [9] under condition $\llbracket \Phi \rrbracket_{2}<1$. In this case, the above algorithm converges to a local minimum of the optimization problem as:

$$
\min _{x}\|y-\Phi x\|_{2}^{2} \quad \text { subject to }\|x\|_{0}<k
$$

\section{B. OMP Algorithm}

Suppose $\mathrm{x}$ is a k sparse signal in $R^{n}$. OMP is one of the greedy methods which is used to solve equation (1). In this method, the measurement matrix $\Phi$ is considered as a dictionary with $\Phi_{i}$ columns which are considered as atoms. Variable $\mathrm{y}(\mathrm{y}=\Phi \times \mathrm{x})$ is a family of $\mathrm{k}$ measurement 
vectors $\left\{y_{1} \ldots \ldots y_{k}\right\}$. This vector is a linear combination from $\mathrm{k}$ column of $\Phi$. Due to reconstruction of the sparse signal we have to identify that which columns of $\Phi$ participate in the measurement vector y [3]. Meanwhile iterations, OMP selects out one atom from the dictionary which minimizes the difference. These atoms are called the residual, between $\mathrm{b}$ and its approximation. Starting iterations with $r_{0}=y$, OMP selects the $\mathrm{k}$-th atom as:

$$
\lambda_{k}=\operatorname{argmax}_{i}=\left|\left\langle r_{k-1}, \Phi_{i}\right\rangle\right| \text {. }
$$

also it updates the residual as:

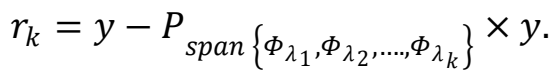

here, $P_{F}$ is the orthogonal projection into the subspace F. experimental results show that the same number of measurements using OMP can provide reconstructions which are compatible to those by BP. However, OMP requires much less execution time in comparison with BP.

\section{The Proposed Method For Tresholding}

In this section we introduce an efficient thresholding method for IHT algorithm that has two follow advantages:

- Using this method, we don't need more than a few sparse component of signal.

- This tresholding method cuse to low complexity in recovery algorithm.

IHT algorithm uses threshold operator $H_{K}(x)$ which selects $\mathrm{k}$ largest coefficient component in magnitude of signal $\mathrm{x}$. In proposed method, we modify threshold operator to $H_{\theta}(x)$ which is:

$$
H_{\theta}(x)=\left\{\begin{array}{lll}
x & \text { if } & |x| \geq \theta \\
0 & \text { if } & |x|<\theta
\end{array}\right.
$$

Meanwhile iterations, we select coefficients which they are greater than a threshold level. Also we decrease threshold value using an exponential function in next iterations. In other hand, threshold value is defined as a follow function:

$$
\theta=\alpha e^{-k \beta}
$$

where $\alpha$ and $\beta$ are constant. Now proposed method can write as a iterative function:

$$
b^{n+1}=H_{\theta}\left[b^{n}+\Phi^{T}\left(y+\Phi \times b^{n}\right)\right]
$$

where $b^{0}=0$. Practically, It is necessary to stop the algorithm after a finite number of iterations. A possible stopping criterion is $\left\|y-\Phi \times b^{n+1}\right\|_{2}<\epsilon$ where $\epsilon=\|e\|_{2}$ where e models possible observation noise due to, for example, sensor noise or quantization errors in digital systems. Also we can select $\alpha$ equal to maximum value in approximated signal $\Phi^{T} \times y$. Also we can select $\beta$ after $n$ iteration threshold value live in under of smaller coefficient which is a decline rate of threshold value.

Hence our proposed method's algorithm (proposed method for Signal Recovery) steps are as follows:

INPUT:

- $\quad$ Obtain $d \times n$ measurement matrix $\Phi$.

- Obtain d-dimensional data vector $y$.
- Maximize minimum component in magnitude of signal $y$.

OUTPUT:

- Estimate $\hat{b}$ in $R^{n}$ for the ideal signal.

\section{PROCEDURE:}

1) Initialize vector $b^{0}=0$, iteration counter $\mathrm{t}=1$, $\alpha=\max (\mathrm{y})$ and $\beta=\mathrm{k}^{*} \min (\mathrm{y}), 0<\mathrm{k}<1$.

2) while the stopping criterion is not met, do $b^{t+1}=H_{\theta}\left[b^{t}+\Phi^{T}\left(y+\Phi \times b^{t}\right)\right]$ then $t=t+1$.

\section{Simulations AND RESUlts}

We used exactly sparse signals of length $\mathrm{N}=512$, with the support of the signal randomly selected from a uniform distribution. The non-zero coefficients were drawn from a standard Gaussian distribution. The signals were measured using a measurement matrix $\Phi$ which satisfy RIP property and $\left\|\Phi_{i}\right\|_{2}<1$. Also, depend on input SNR, we added arbitrary white noise with measurement vector $y=\Phi \times x+e$. Simulating all explained methods attendant our proposed method resulted follow figures and tables. All results are average over 100 iterations.

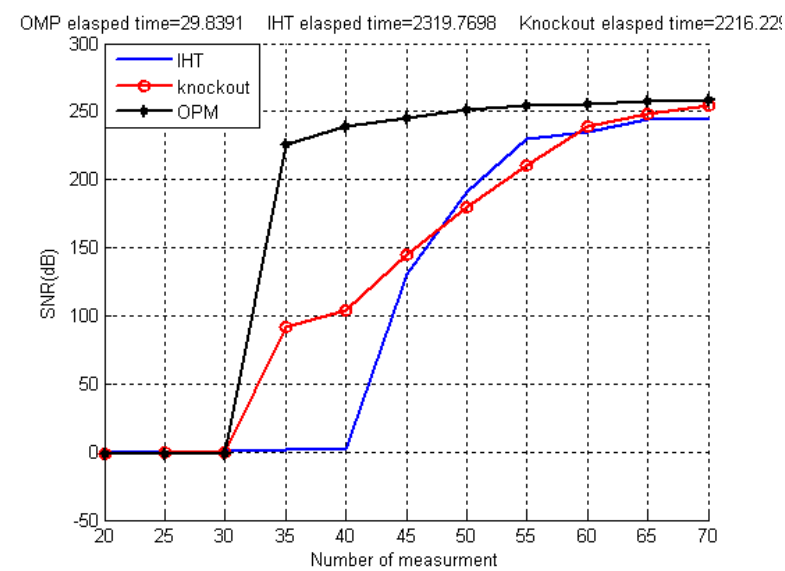

Fig. 1. SNR of reconstructed sparse signal. Nonzero coefficient $=10$ iteration number $=700$

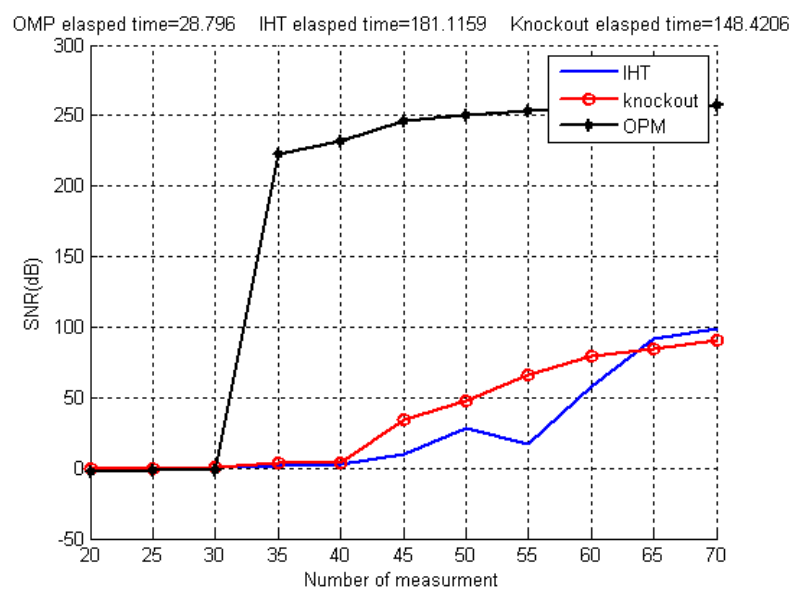

Fig. 2. SNR of reconstructed sparse signal. Nonzero coefficient=10 iteration number $=200$

Initially we generated sparse signal with $\mathrm{SNR}=250 \mathrm{dBm}$. Then we started to measure using a measurement matrix $\Phi$ under varying number of measurements. Then we executed OMP, IHT and proposed recovery algorithms with the data 
vector $y=\Phi \times x+e$. For first stage we generated sparse signal with 10 nonzero coefficients. Fig. 1 and Fig. 2 illustrates the SNR results of reconstructed signal in 700 iterations and 200 iterations respectively.

Fig. 1 and 2 indicate that IHT and Knockout algorithms are appropriate algorithms for small number of nonzero coefficients. For same results in comparison with OMP, we must run IHT and Knockout with more iteration which accrues to high executed time. For small number of iterations, the performance of IHT and Knockout are less than OMP. Table I and Table II result about recovery performance of mentioned algorithms for sparse signal with 10 nonzero components.

TABLE I: SNR AND ELAPSED TIME FOR RECOVERY SIGNAL OF LENGTH512 AND SPARSITY EQUAL TO 10, NUMBER OF MEASUREMENT $=50$.

\begin{tabular}{llll}
\hline \multicolumn{2}{l}{ Nonzero component=10 } & number of measurement=40 \\
\hline \multirow{2}{*}{ OMP } & SNR(dB) & $\begin{array}{l}\text { Elapsed } \\
\text { time(s) }\end{array}$ & $\begin{array}{l}\text { Iteration } \\
\text { number }\end{array}$ \\
\cline { 2 - 4 } & 248.01 & 3.17 & --------- \\
\hline IHT & 19.20 & 3.47 & -------- \\
\hline $\begin{array}{l}\text { Proposed } \\
\text { method }\end{array}$ & 48.98 & 73.02 & 350 \\
\hline & 65.38 & 329.67 & 700 \\
\hline
\end{tabular}

TABLE II: SNR AND ELAPSED TIME FOR RECOVERY SIGNAL OF LENGTH512 AND SPARSITY EQUAL TO 10, NUMBER OF MEASUREMENT=50

\begin{tabular}{llll}
\hline Nonzero component=10 & number of measurement=50 \\
\hline \multirow{2}{*}{ OMP } & SNR(dB) & $\begin{array}{l}\text { Elapsed } \\
\text { time(s) }\end{array}$ & $\begin{array}{l}\text { Iteration } \\
\text { number }\end{array}$ \\
\cline { 2 - 4 } & 252 & 4.14 & --------- \\
\hline IHT & 85.77 & 4.09 & -------- \\
\hline $\begin{array}{l}\text { Proposed } \\
\text { method }\end{array}$ & 192.89 & 76.14 & 350 \\
\cline { 2 - 4 } & 200.82 & 328.4 & 700 \\
\hline
\end{tabular}

TABLE III: SNR AND ELAPSED TIME FOR RECOVERY SIGNAL OF LENGTH512 AND SPARSITY EQUAL TO 100, NUMBER OF MEASUREMENT $=300$

\begin{tabular}{|c|c|c|c|}
\hline \multicolumn{2}{|c|}{$\begin{array}{l}\text { Nonzero component }=100 \\
\text { measurement }=300\end{array}$} & \multicolumn{2}{|r|}{ number of } \\
\hline & $\mathrm{SNR}(\mathrm{dB})$ & $\begin{array}{l}\text { Elapsed } \\
\text { time(s) }\end{array}$ & $\begin{array}{l}\text { Iteration } \\
\text { number }\end{array}$ \\
\hline \multirow[t]{2}{*}{ OMP } & 253.09 & 414.03 & --------- \\
\hline & 253.29 & 432.15 & ---------- \\
\hline \multirow[t]{2}{*}{ IHT } & 254.02 & 144.47 & 200 \\
\hline & 254.07 & 212.27 & 300 \\
\hline \multirow{2}{*}{$\begin{array}{l}\text { Proposed } \\
\text { method }\end{array}$} & 217.16 & 84.53 & 200 \\
\hline & 253.94 & 118.69 & 300 \\
\hline
\end{tabular}

In this stage we performed above step for sparse signal with 100 nonzero coefficients. Fig.3 illustrates SNR of recovered signal with OMP is higher than IHT and Knockout but executed time for OMP and IHT is very more than Knockout method. Then we added additive Gaussian noise to achieve input SNR of $50 \mathrm{~dB}$ and performed the stage one. Fig.4 shows that each three algorithms have same performance but Knockout method has lower executed times. Table III and Table IV show some results about recovery performance of above algorithms for sparse signal with 10 nonzero components.
TABLE IV: SNR AND ELAPSED TIME FOR RECOVERY SIGNAL OF LENGTH512 AND SPARSITY EQUAL TO 100, NUMBER OF MEASUREMENT $=250$

\begin{tabular}{|c|c|c|c|}
\hline \multicolumn{2}{|c|}{$\begin{array}{l}\text { Nonzero component }=100 \\
\text { measurement }=250\end{array}$} & \multicolumn{2}{|r|}{ number of } \\
\hline & $\mathrm{SNR}(\mathrm{dB})$ & $\begin{array}{l}\text { Elapsed } \\
\text { time(s) }\end{array}$ & $\begin{array}{l}\text { Iteration } \\
\text { number }\end{array}$ \\
\hline \multirow[t]{2}{*}{ OMP } & 238.76 & 305.6 & ---------- \\
\hline & 238.16 & 301.4 & --------- \\
\hline \multirow[t]{2}{*}{ IHT } & 177.55 & 178.41 & 200 \\
\hline & 243.92 & 124.95 & 300 \\
\hline \multirow{2}{*}{$\begin{array}{l}\text { Proposed } \\
\text { method }\end{array}$} & 138.05 & 65.14 & 200 \\
\hline & 190.28 & 94.84 & 300 \\
\hline
\end{tabular}

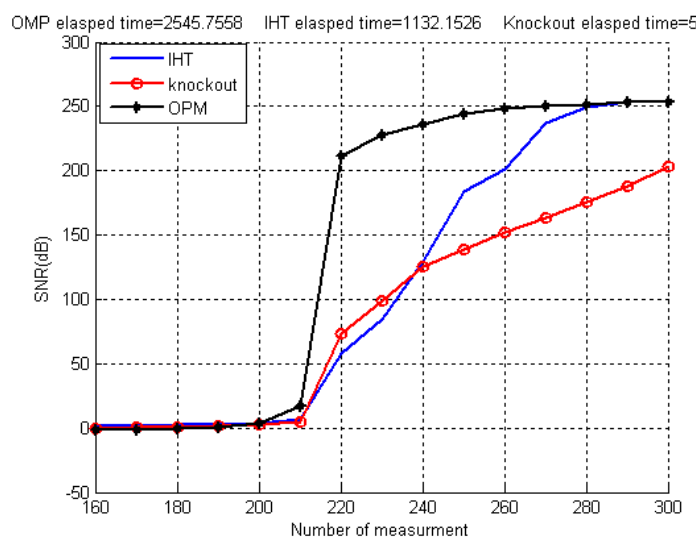

Fig. 3. SNR of reconstructed sparse signal. Nonzero coefficient $=100$ iteration number $=200$

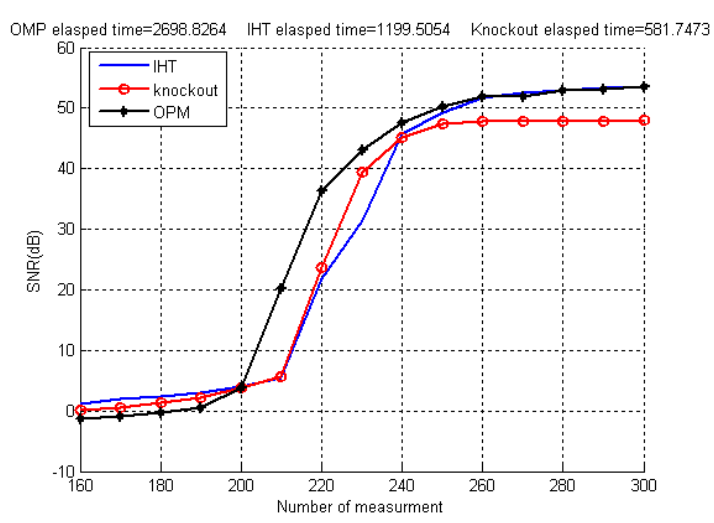

Fig. 4. SNR of reconstructed sparse signal. Nonzero coefficient $=100$ iteration number $=200$, input $\mathrm{SNR}=50 \mathrm{dBm}$

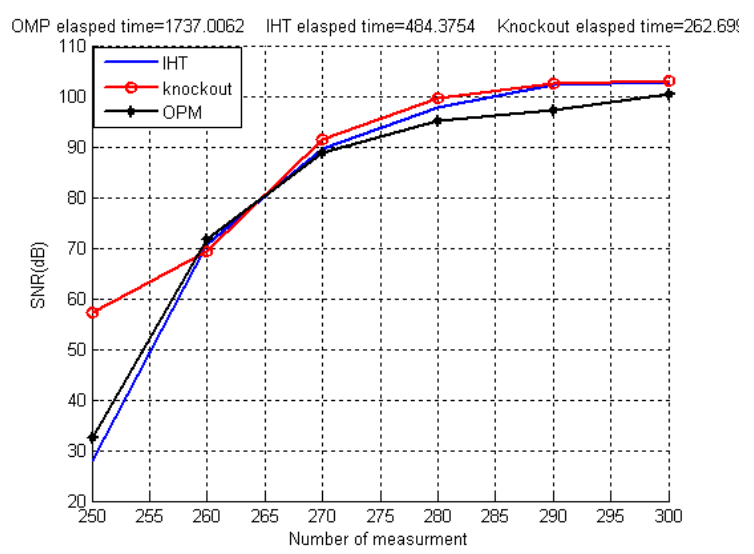

Fig. 5. SNR of reconstructed sparse signal. Nonzero coefficient $=100$ iteration number $=150$, input $\mathrm{SNR}=100 \mathrm{dBm}$

Not bad we mention here that whatever nonzero components in sparse signal increase the measurements 
number must be increase for exact reconstruction and Knockout perform recovery role better. For more intuition we performed recovery process with 120 nonzero components in sparse signal. We used 250 to 300 measurements and 150 iterations for IHT and Knockout methods. Fig.5 indicates that Knockout method has same performance with IHT and OMP method but Knockout is faster.

\section{CONCLUSION}

The performance of greedy algorithms is examined in this work. Greedy algorithms are more practicable than BP recovery algorithms because of greedy algorithms is faster than BP algorithms. In this paper, a novel approach of thresholding in greedy algorithm was proposed. This thresholding led to low complexity computational in IHT. In large scale signal where we have greater than \%15 sparsity, this method is appropriate due to fast recovery. In this paper simulation results on variety of sparsity showed that Knockout method has performance as same as OMP and IHT but Knockout method is very faster than other two algorithms and can recover exact signals in much less time.

\section{REFERENCES}

[1] E. J. Candes, J. Romberg, and T. Tao, "Robust uncertainty principles: exact signal reconstruction from highly incomplete frequency information," IEEE Transactions on Information Theory, vol. 52, no. 2, pp. 489-509, 2006.

[2] D. L. Donoho, "Compressed sensing," IEEE Transactions on Information Theory, vol. 52, no. 4, pp. 1289-1306, 2006.

[3] J. Tropp and A. Gilbert, "Signal recovery from partial information via orthogonal matching pursuit," IEEE Trans. Inform. Theory, vol. 53, no. 12, pp. 4655-4666, Dec. 2007.
[4] Candes, E. J.; Tao, T, "Decoding by linear programming," IEEE Trans. Inform. Theory, vol. 51, no. 12, pp. 4203-4215, Dec. 2005.

[5] G. Davis, S. Mallat, and M. Avellaneda, "Greedy adaptive approximation," J. Constr. Approx. 13, pp. 57-98, 1997.

[6] A. C. Gilbert, M. Muthukrishnan, and M. J. Strauss, "Approximation of functions over redundant dictionaries using coherence," in Proc. $14^{\text {th }}$ Апnи. ACM-SIAM Symp. Discrete Algorithms, Baltimore, MD, Jan. 2003, pp. 243-252.

[7] S. S. Chen, D. L. Donoho, and M. A. Saunders, "Atomic decomposition by basis pursuit," SIAM J. Sci. Comput. vol. 20, no. 1, pp. 33-61, 1999.

[8] T. Blumensath and M. Davies, "Iterative thresholding for sparse approximations", Journal of Fourier Analysis and Applications vol. 14, no. 5, 2008, pp. 629-654.

[9] R. Baraniuk, M. Davenport, R. DeVore, and M. B. Wakin, "The johnson-lindenstrauss lemma meets compressed sensing," http://www.dsp.rice.edu/cs/jlcs-v03.pdf, May 2006.

[10] Lei Zhu, Yaolin Zhu, Huan Mao, and Meihua Gu, "A New Method for Sparse Signal Denoising Based on Compressed Sensing," KAM 2009, vol. 1 , pp. $35-38$.

[11] E. Candes and T. Tao, "Near-optimal signal recovery from random projections: universal encoding strategies," IEEE Trans. Inform. Theory, vol. 52, pp. 5406-5425, 2004.

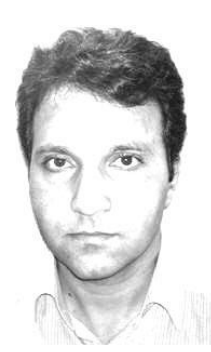

Ali Pourmohammad was born in Azerbaijan. He has a Ph.D. in Electrical Engineering (Signal Processing) from the Electrical Engineering Department, Amirkabir University of Technology, Tehran, Iran. He also teaches several courses (C++ programming, multimedia systems, Microprocessor Systems, digital audio processing, digital image processing and digital signal processing I and II). His research interests include digital signal processing and applications (audio and speech processing and applications, digital image processing and applications, sound source localization, sound source separation (determined and under-determined blind source separation (BSS), Audio, Image and Video Coding, Scene Matching and ...) and multimedia and applications. 